\title{
Bi-directional electrons in the near-Earth plasma sheet
}

\author{
K. Shiokawa ${ }^{1}$, W. Baumjohann ${ }^{2}$, and G. Paschmann ${ }^{3}$ \\ ${ }^{1}$ Solar-Terrestrial Environment Laboratory, Nagoya University, Toyokawa 442-8507, Japan \\ ${ }^{2}$ Institut für Weltraumforschung der Österreichischen Akademie der Wissenschaften, 8042 Graz, Austria \\ ${ }^{3}$ Max-Planck-Institut für extraterrestrische Physik, 85740 Garching, Germany
}

Received: 4 February 2002 - Revised: 30 December 2002 - Accepted: 21 February 2003

\begin{abstract}
We have studied the occurrence characteristics of bi-directional electron pitch angle anisotropy (enhanced flux in field-aligned directions, $F_{\|} / F_{\perp}>1.5$ ) at energies of $0.1-30 \mathrm{keV}$ using plasma and magnetic field data from the AMPTE/IRM satellite in the near-Earth plasma sheet. The occurrence rate increases in the tailward direction from $X_{\mathrm{GSM}}=-9 R_{E}$ to $-19 R_{E}$. The occurrence rate is also enhanced in the midnight sector, and furthermore, whenever the elevation angle of the magnetic field is large while the magnetic field intensity is small, $B \sim 15 \mathrm{nT}$. From these facts, we conclude that the bi-directional electrons in the central plasma sheet are produced mainly in the vicinity of the neutral sheet and that the contribution from ionospheric electrons is minor. A high occurrence is also found after earthward high-speed ion flows, suggesting Fermi-type fieldaligned electron acceleration in the neutral sheet. Occurrence characteristics of bi-directional electrons in the plasma sheet boundary layer are also discussed.
\end{abstract}

Key words. Magnetospheric physics (magnetospheric configuration and dynamics; magnetotail; plasma sheet)

\section{Introduction}

Bi-directional electron pitch angle anisotropy is commonly observed in the nightside magnetosphere (e.g. Klumpar, 1993 and references therein). Hada et al. (1981) were the first to analyze the field-aligned bi-directional electrons systematically at radial distances of $\sim 8-30 R_{E}$ using data from the IMP-6 satellite. They concluded that the bi-directional electrons are observed near the neutral sheet, suggesting their formation by a Fermi acceleration process. Smets et al. (1999) have compared observation and model calculation of three types (beam-like, conic-like, and perpendicularly peaked) of electron distributions, and concluded that the (parallel) Fermi acceleration is the leading process com-

Correspondence to: K. Shiokawa

(shiokawa@stelab.nagoya-u.ac.jp) pared to (perpendicular) betatron heating at the dipolarization phase of a substorm. Sergeev et al. (2001) have reported the association of bi-directional electrons with the near-Earth flux transport related to plasma bubbles, on the basis of observations at several outbound passes by the Active Magnetospheric Particle Tracer Explorer/Ion Release Module (AMPTE/IRM) satellite. However, detailed statistics of the occurrence of bi-directional electrons at radial distances outside $10 R_{E}$ have not been investigated yet, except for a study done by Sugiyama et al. (1997) for magnetically quiet intervals.

Since the study of bi-directional electrons by Hada et al. (1981), there have been several important findings regarding high-speed ion flows in the near-Earth plasma sheet at $10-30 R_{E}$. One is the existence of the plasma sheet boundary layer (PSBL) (e.g. Eastman et al., 1984, 1985; Takahashi and Hones, 1988; Baumjohann et al., 1988). The other is the observation of high-speed ion flow (bursty bulk flow) near the neutral sheet (Baumjohann et al., 1990; Angelopoulos et al., 1992, 1994). The statistical relations of the occurrence of bi-directional electrons to these ion flow structures have not been investigated yet.

In this paper, we investigate the occurrence characteristics of the bi-directional electrons with a parallel and perpendicular flux ratio of more than 1.5 in the plasma sheet at $\left|X_{\mathrm{GSM}}\right|=9-19 R_{E}$ using data obtained by the AMPTE/IRM satellite. From the observed characteristics, we conclude that the bi-directional electrons at these distances are produced mainly by processes in the vicinity of the neutral sheet and that ionospheric electrons are a minor source for bi-directional electrons in the plasma sheet. The occurrence characteristics in relation to the ion flow structures and magnetic activities are also reported.

\section{Data and examples}

In the present analysis we use data from the magnetic field instrument (Lühr et al., 1985) and the three-dimensional 
(3-D) plasma analyzer (Paschmann et al., 1985) on board the AMPTE/IRM satellite. The satellite measures 3-D plasma distribution every $4.35 \mathrm{~s}$. Due to a limitation in the data transmission rate, however, not all 3-D distributions are transmitted to the ground. The time resolution of the 3-D distribution varies between $\sim 4-40$ s, depending on the transmission mode.

To define the field-aligned bi-directional electrons, we calculate parallel and perpendicular energy fluxes of electrons in units of $\mathrm{cm}^{-2} \mathrm{~s}^{-1} \mathrm{sr}^{-1}$ by integrating electron fluxes for energies of $0.1-30 \mathrm{keV}$ and for pitch angles of $0^{\circ}-15^{\circ}\left(F_{\|, 1}\right)$, $165^{\circ}-180^{\circ}\left(F_{\|, 2}\right)$, and $75^{\circ}-105^{\circ}\left(F_{\perp}\right)$. In order to avoid the contamination of electron fluxes due to photoelectrons from the satellite, the fluxes with energies below $0.1 \mathrm{keV}$ are omitted from the present analysis. We define the field-aligned bi-directional electrons as the events which satisfy the conditions (1) $F_{\|} / F_{\perp}>1.5$ where, $F_{\|}=\left(F_{\|, 1}+F_{\|, 2}\right) / 2$ and (2) $F_{\|, 1} / F_{\|, 2}<1.5$ and $F_{\|, 2} / F_{\|, 1}<1.5$. It should be noted that by integrating over the entire energy range, we omit cases of multi-component distributions, e.g. those where low energy electrons show field-aligned bi-directional features, while the higher energies show trapped features. Our analysis in this paper focuses on bi-directionality in the major component of electron energy fluxes.

The plasma sheet data set and the definitions used in the present analysis are the same as those used by Baumjohann et al. $(1988,1989)$. The AMPTE/IRM data in the near-Earth tail $\left(X_{\mathrm{GSM}}=-9\right.$ to $-19 R_{E}$ and $\left.\left|Y_{\mathrm{GSM}}\right|<15 R_{E}\right)$ are divided into lobe, plasma sheet boundary layer (PSBL), and central plasma sheet (CPS) regions. The lobe is defined as the region where no electrons and ions in $\mathrm{keV}$ energy range are observed, that is, the region where the partial densities of $1.8-30 \mathrm{keV}$ electrons and $8.5-40 \mathrm{keV}$ ions are less than 2count levels $\left(1 \times 10^{-4}\right.$ and $2 \times 10^{-3} \mathrm{~cm}^{-3}$, respectively). By checking for photoelectrons in the low-density regions, the rest of the data is divided into CPS and PSBL. More exactly, CPS and PSBL are defined as the regions of $n_{e}<0.94 \cdot n_{p}^{0.86}$ and $n_{e}>0.94 \cdot n_{p}^{0.86}$, where $n_{e}$ and $n_{p}$ denote measured electron and ion densities including low-energy photoelectrons, respectively. This criterion of photoelectrons is rather useful to define the difference in plasma regimes in CPS and PSBL, as indicated by Fig. 5 of Baumjohann et al. (1988). The CPS data are further divided into inner and outer parts by using horizontal magnetic field intensity. The inner CPS is defined as the region closer to the neutral sheet with weak magnetic fields $\left(\left(B_{x}^{2}+B_{y}^{2}\right)^{1 / 2}<15 \mathrm{nT}\right)$.

Figure 1 shows an example of the bi-directional electrons which were observed by AMPTE/IRM on 8 April 1985. During the plotted interval, a large substorm was in progress, with a maximum $A E$ index of 10:43 nT at 23:23 UT. The satellite was mostly in the inner CPS, close to the neutral sheet at $X_{\mathrm{GSM}} \sim 14 R_{E}$. Note that the vertical distance $Z_{N S}$ from the model neutral sheet (Fairfield, 1980) was less than $0.2 R_{E}$ for the plotted interval. Several bursts of highspeed ion flows were observed with velocities higher than $400 \mathrm{~km} / \mathrm{s}$ at 23:07-23:09 UT and 23:18-23:23 UT. The flow vectors were mostly directed earthward for the plotted interval. It is clearly seen that intense bi-directional electrons with a flux ratio $\left(F_{\|} / F_{\perp}\right)$ of more than 1.5 were observed after the passage of the high-speed flows. The bi-directional electrons were also observed during the high-speed flow, but the flux ratio was smaller than that after the high-speed flow. Another clear example of an intense bi-directional electrons after the passage of high-speed flow was shown in Plate 1 by Shiokawa et al. (1998).

Figure 2 shows another example of bi-directional electrons in the inner CPS observed on 7 April 1985. The satellite was continuously in the inner CPS under fairly quiet conditions without any flows. The $A E$ index was less than $60 \mathrm{nT}$ for $1 \mathrm{~h}$ before and during the plotted interval. At $\sim 01: 10 \mathrm{UT}$, the electron energy suddenly decreased to less than $1 \mathrm{keV}$, while ion energy was a constant. Intense bi-directional electrons with flux ratios of more than 4 were observed in this electron transition region. This event is similar to the quiettime highly-collimated electrons reported by Sugiyama et al. (1997). Although the cause of this type of event is not clear, it may be a crossing of the magnetospheric source region of quiet-time auroral arc.

Figure 3 shows the bi-directional electron example which was observed in the vicinity of the PSBL on 30 March 1985. The satellite was mostly in the PSBL for 00:54-01:00 UT where low-energy photoelectrons below $0.1 \mathrm{keV}$ were observed. The electrons with energies above $0.1 \mathrm{keV}$ show a field-aligned bi-directional feature with flux ratios of 1.52.5. Then the satellite observed an earthward ion flow along the field line for 00:59-01:06 UT. This flow is a typical feature in the PSBL (Takahashi and Hones, 1988). Just after this flow, the satellite observed intense bi-directional electrons twice (01:07-01:10 UT and 01:16-01:20 UT).

\section{Statistical analysis}

Using data from the AMPTE/IRM satellite for FebruaryMay, 1985 and March-June, 1986, we examine the dependence of the bi-directional electron occurrence on different plasma sheet regions, magnetic activity, local magnetic field, and high-speed ion flow.

Figures $4 \mathrm{a}$ and $\mathrm{b}$ show the coverage of the AMPTE/IRM orbits used in the analysis in the $X_{\mathrm{GSM}}-Y_{\mathrm{GSM}}$ and $X_{\mathrm{GSM}}-$ $Z_{N S}$ plane, respectively. The orbit covers $-19<X_{\mathrm{GSM}}<$ $-9 R_{E},-13<Y_{\mathrm{GSM}}<+13 R_{E}$, and $-10<Z_{N S}<$ $+2 R_{E}$. In these orbits 386313 -D plasma distributions (4.35 s samples) in total were obtained. By the definitions described in the previous section, 8021 bi-directional electron events were selected from the 3-D plasma distributions. The occurrence rate of bi-directional electrons was defined by normalizing the number of bi-directional electron distributions (events) by the total number of distribution samples in the relevant regions. 


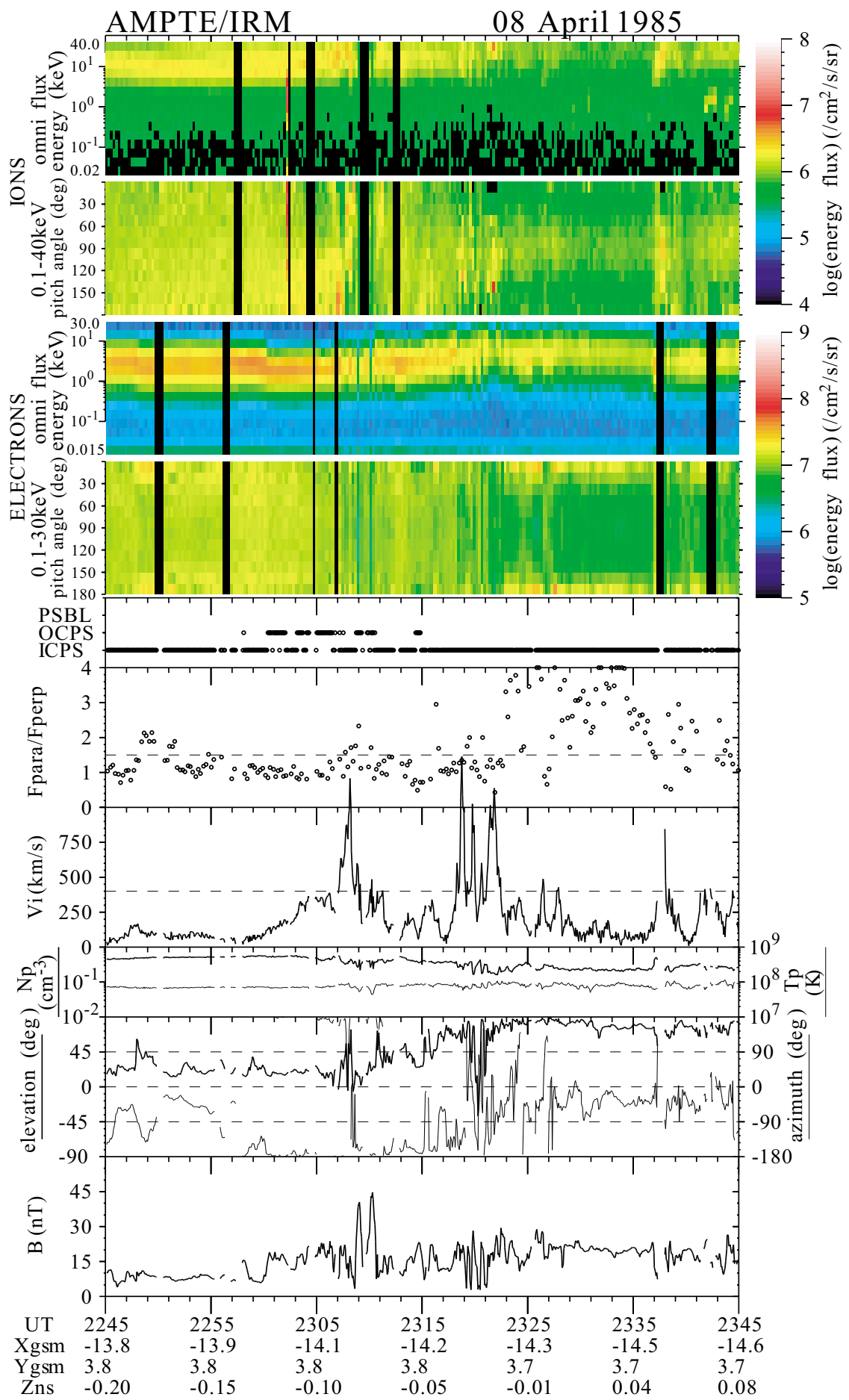

Fig. 1. Example of field-aligned bi-directional electrons after the passage of earthward high-speed ion flow in the central plasma sheet. From top to bottom: energy-time spectra of omni-directional ions, pitch angle-time spectra of ions for energies of $0.1-40 \mathrm{keV}$, energy-time spectra of omni-directional electrons, pitch angle-time spectra of electrons for energies of $0.1-30 \mathrm{keV}$, plasma sheet regions where the satellite was located, ratio of parallel and perpendicular electron energy fluxes, ion flow velocity, density (thick line) and temperature (thin line) of ions, elevation (thick line) and azimuth (thin line) of the magnetic field in GSM coordinates, and magnetic field intensity, measured on board the AMPTE/IRM satellite for 22:45-23:45 UT on 8 April 1985. The plasma sheet regions are defined by Baumjohann et al. (1988, 1989) where PSBL, OCPS, and ICPS are plasma sheet boundary layer, outer central plasma sheet, and inner central plasma sheet, respectively. The parallel electron energy flux is the average total energy flux (for energies of $0.1-40 \mathrm{keV}$ ) at pitch angles of $0^{\circ}-15^{\circ}$ and $165^{\circ}-180^{\circ}$, while the perpendicular energy flux is calculated for pitch angles of $75^{\circ}-105^{\circ}$. Satellite locations in units of Earth radii are shown at the bottom of these panels in GSM- $X Y$ coordinates, while $Z_{N S}$ is the vertical distance from the model neutral sheet by Fairfield (1980). 


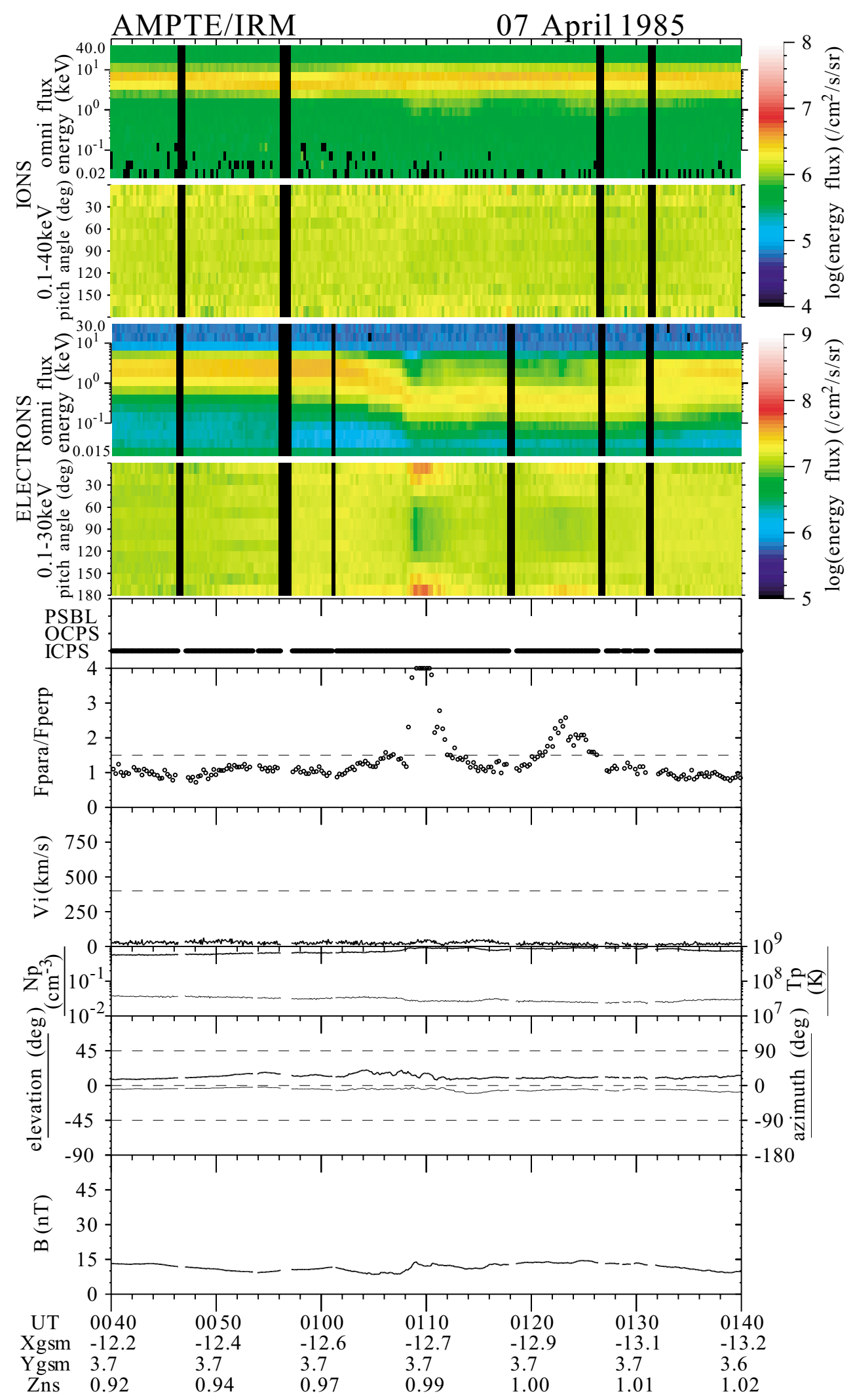

Fig. 2. Example of field-aligned bi-directional electrons observed during magnetically quiet interval in the central plasma sheet for 00:4001:40 UT on 7 April 1985. The format is the same as that of Fig. 1. 


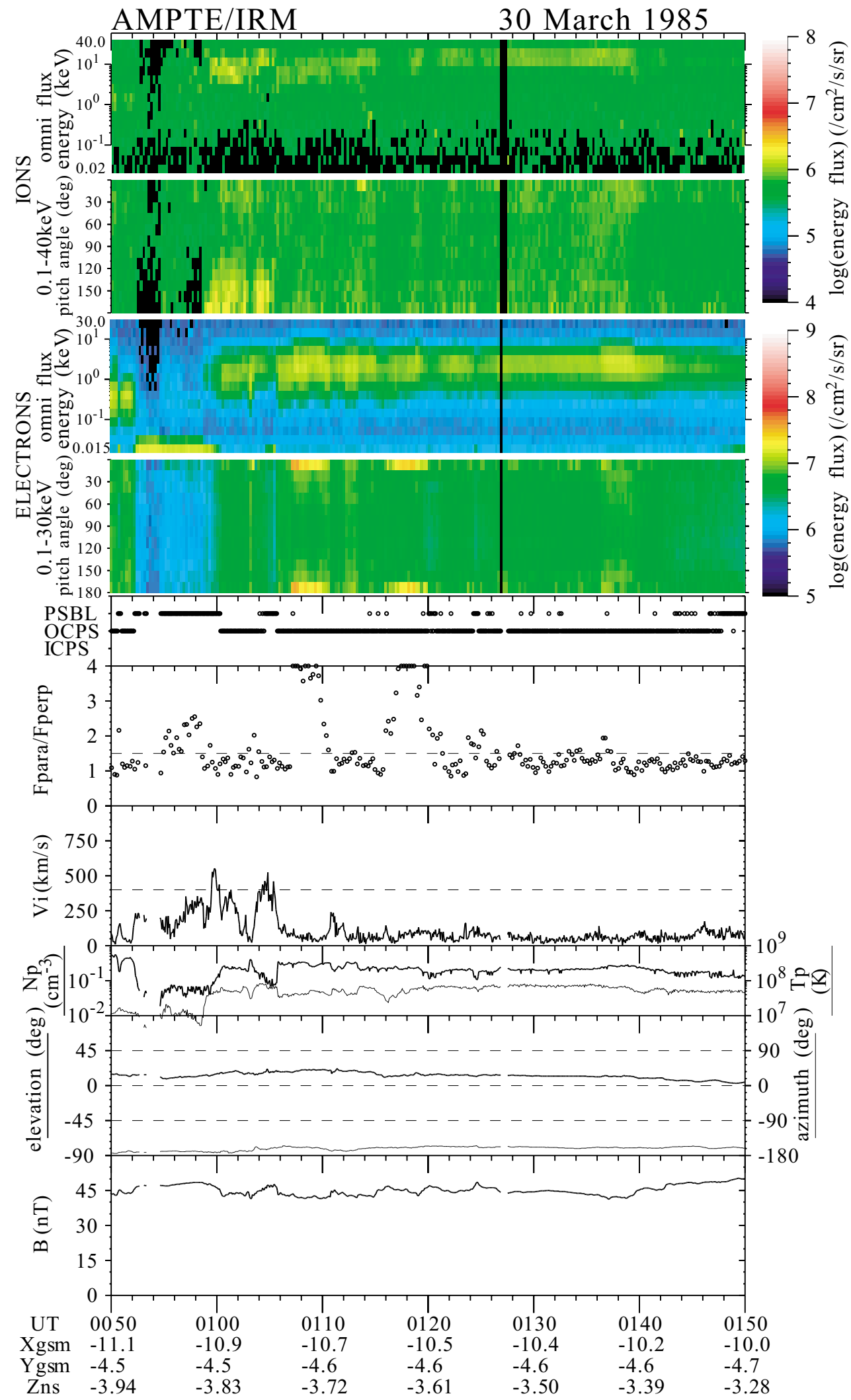

Fig. 3. Example of field-aligned bi-directional electrons observed in the vicinity of the plasma sheet boundary layer for 00:50-01:50 UT on 30 March 1985. The format is the same as that of Fig. 1. 

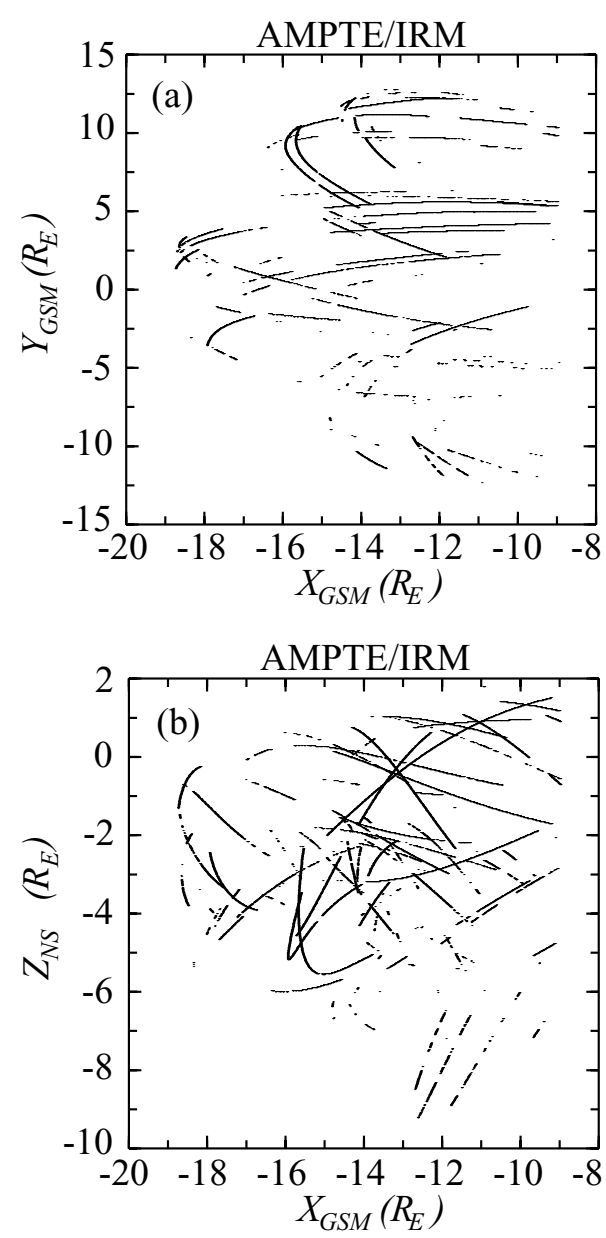

Fig. 4. AMPTE/IRM orbits in (a) GSM- $X Y$ and (b) GSM- $X-Z_{N S}$ planes used in the present analysis.

\subsection{Plasma sheet regions}

As shown in the examples of Figs. 1-3, bi-directional electrons are observed in various plasma sheet regions. Figure 5 shows the occurrence rate, as well as the energies and fluxes of the bi-directional electrons at different plasma sheet regions. According to the definition by Baumjohann et al. $(1988,1989)$, we divided the plasma sheet into the regions of inner CPS, outer CPS, CPS within 3 min before and after the PSBL entry, and PSBL. The satellite is located closest to the neutral sheet in the inner CPS and most distant from the neutral sheet at the PSBL.

As shown in the top panel of Fig. 5, the occurrence rate is highest in the inner CPS. It decreases toward the PSBL but has a second peak in the outermost layers of the CPS, within 3 min to the PSBL entry. Both the parallel energy and flux decrease from the inner CPS to the PSBL. These energy and flux changes are consistent with the change in the background plasma from the neutral sheet to the lobe.

In the following analysis, we redefine the CPS to include both the inner and outer CPS and the PSBL to include both the PSBL and the CPS within 3 min before and after the

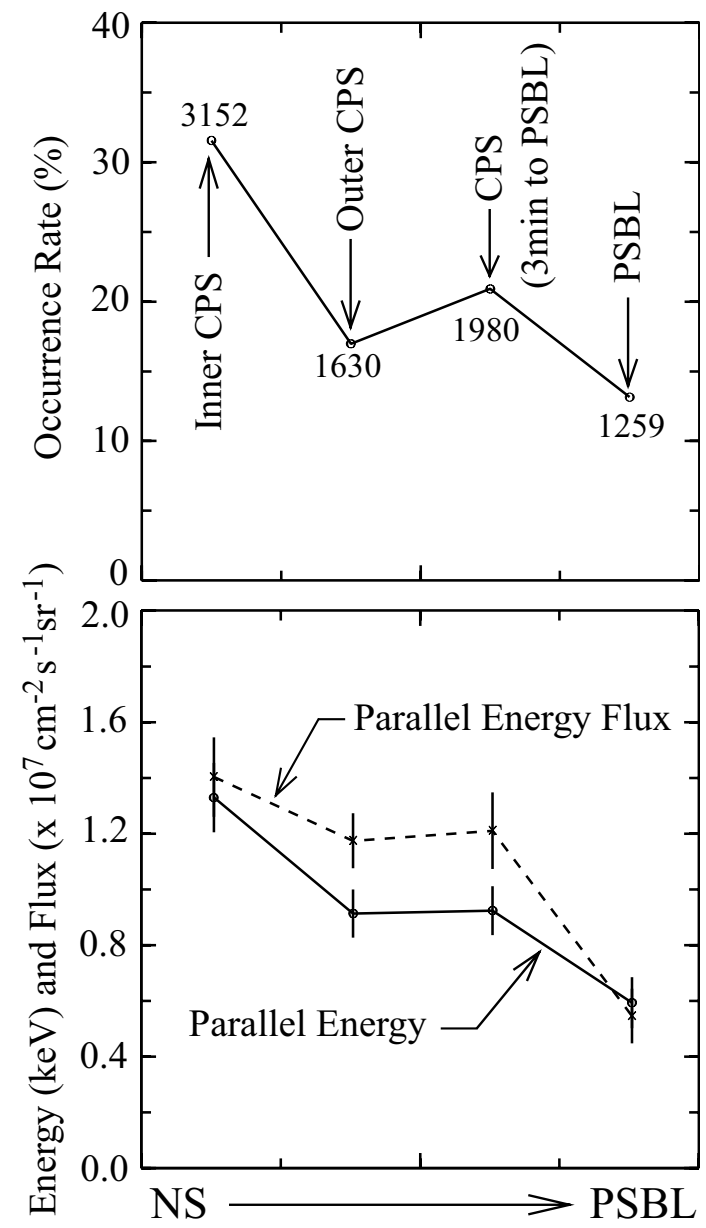

Fig. 5. (Top) occurrence rate and (bottom) parallel energy (solid line) and parallel energy flux (dashed line) of bi-directional electrons at four different plasma sheet regions where CPS and PSBL are central plasma sheet and plasma sheet boundary layer, respectively. From left to right, the satellite leaves from the neutral sheet and approaches to the lobe. The numbers of bi-directional electron events used for the calculation of the occurrence rate are shown in the top panel. Error bars in the bottom panel represent $\pm 0.2 \sigma$.

PSBL entry. The numbers of bi-directional electron events are 4782 and 3239 in the newly-defined CPS and PSBL, respectively.

Figures $6 \mathrm{a}$ and $\mathrm{b}$ show $X_{\mathrm{GSM}}$ and $Y_{\mathrm{GSM}}$ dependences of bidirectional electron occurrence, respectively. It is a surprising result that the occurrence rate in the CPS increases tailward and reaches up to $50 \%$ in the region close to $X_{\mathrm{GSM}}=$ $-19 R_{E}$. This fact indicates that bi-directional electrons by our definition $\left(F_{\|} / F_{\perp}>1.5\right)$ are quite a common feature in this tail region. A high occurrence rate in the CPS is also observed around midnight $\left(Y_{\mathrm{GSM}}=0\right)$. For the occurrence in the PSBL, a weak midnight preference is seen, while the $X_{\mathrm{GSM}}$ dependence is not clear. 

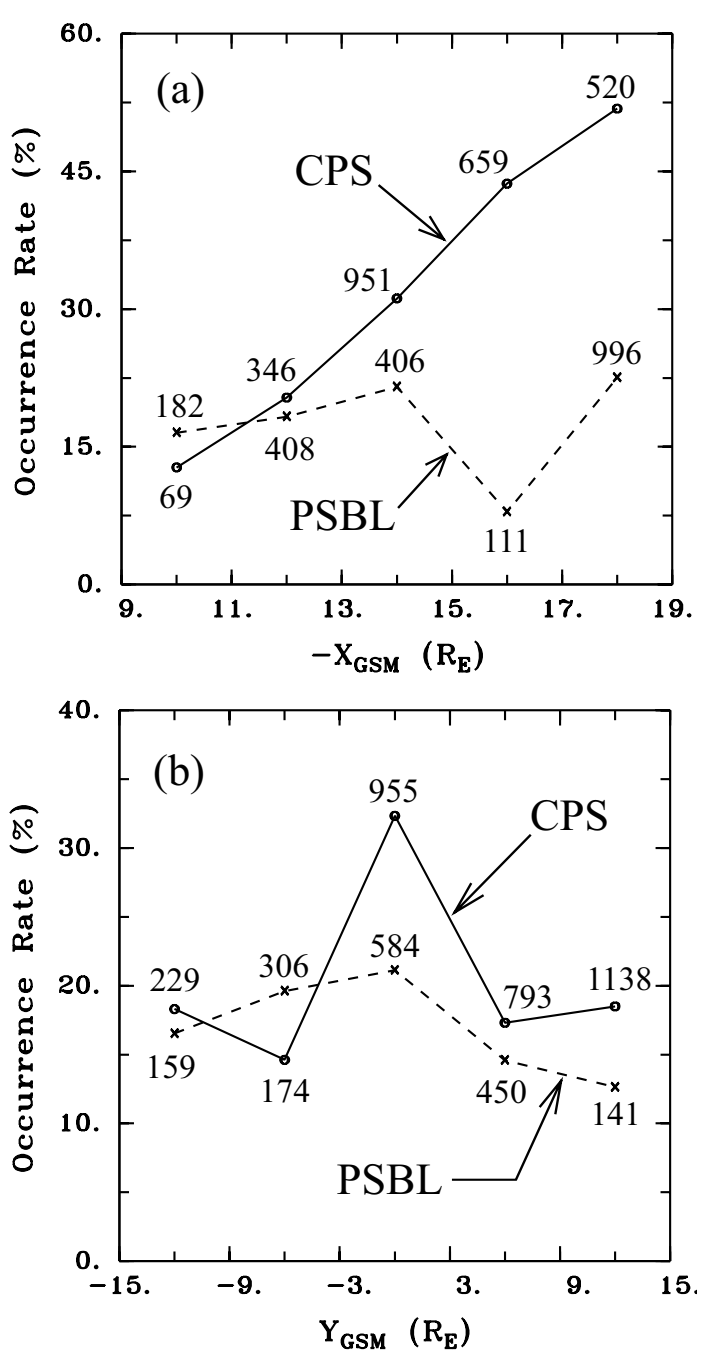

Fig. 6. Occurrence rate of bi-directional electrons in the central plasma sheet (solid line) and the plasma sheet boundary layer (dashed line) in (a) $X_{\mathrm{GSM}}$ and (b) $Y_{\mathrm{GSM}}$ directions. Data from the midnight sector plasma sheet $\left(\left|Y_{\mathrm{GSM}}\right|<5 R_{E}\right)$ are used in panel (a), while data from the near-Earth region $\left(\left|X_{\mathrm{GSM}}\right|<15 R_{E}\right)$ are used in panel (b), because of the limitation of orbital coverage shown in Fig. 4. The number of bi-directional electron events used for the calculation of the occurrence rate is given in the top panel.

\subsection{Magnetic activity}

Figure 7 shows the dependence of occurrence, energy, and flux of bi-directional electrons on the $A E$ index, which indicates magnetic activity in the auroral zone. In both the CPS and the PSBL regions, the occurrence rate and the parallel energy increase as magnetic activity goes up. The occurrence rate seems to saturate for $A E>300 \mathrm{nT}$. The dependence of energy flux on the $A E$ index is not clear in the CPS region, while it increases for large $A E$ in the PSBL region.

\subsection{Local magnetic field}

In order to see the relation between the bi-directional electrons and the neutral sheet, we have checked the dependence

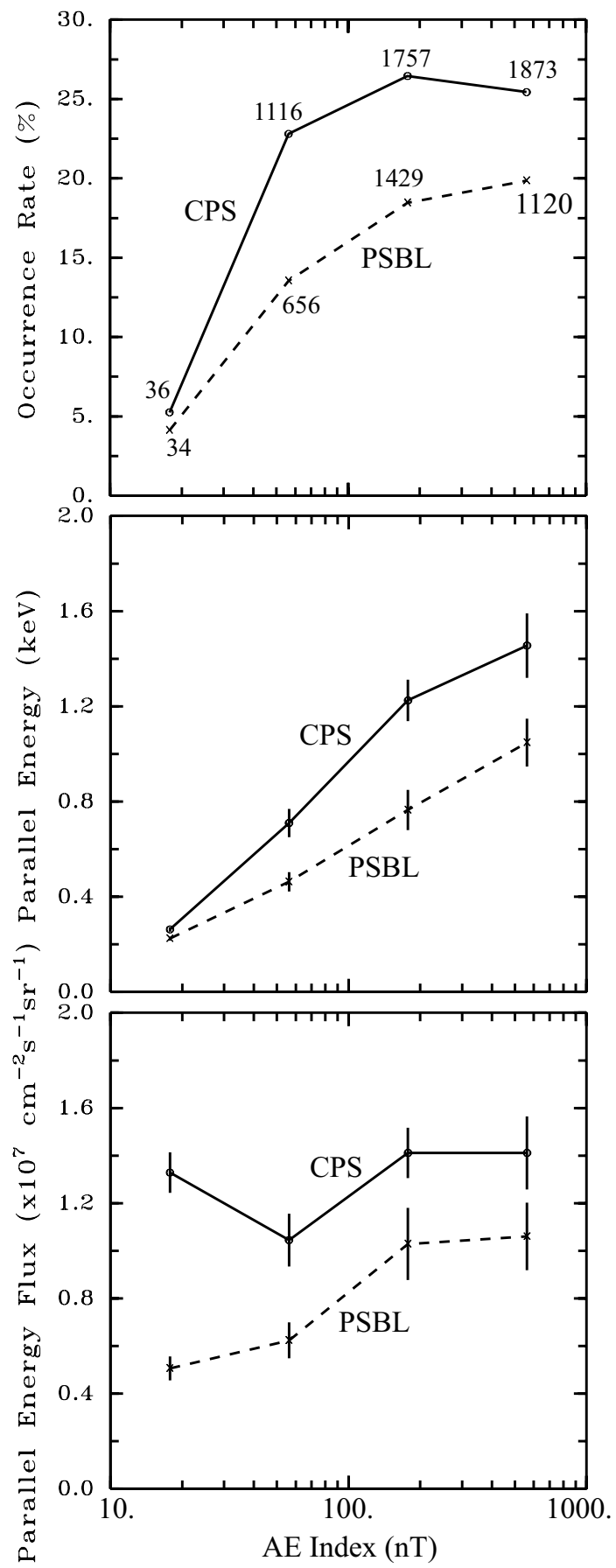

Fig. 7. From top to bottom, occurrence rate, parallel energy, and parallel energy flux of bi-directional electrons for different values of the $A E$ index in the central plasma sheet (solid line) and plasma sheet boundary layer (dashed line). The number of bi-directional electron events used for the calculation of the occurrence rate is given in the top panel. Error bars in the bottom two panels represent $\pm 0.2 \sigma$.

of bi-directional electron occurrence on the intensity, $B$, and the elevation angle, $\theta$, of the local magnetic field. Note that in the neutral sheet, $B$ becomes minimum and $\theta$ becomes $90^{\circ}$. Figure 8 shows the dependence on the elevation angle. 


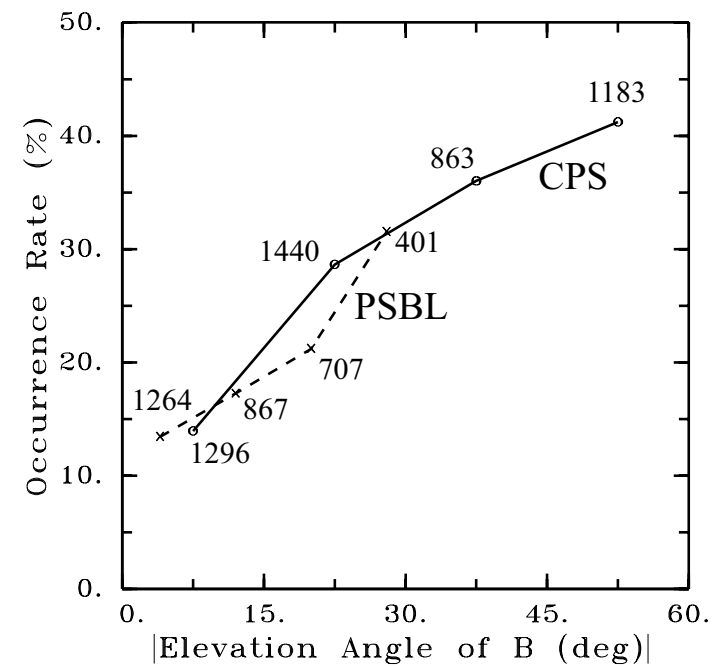

Fig. 8. Occurrence rate of bi-directional electrons at different elevation angles of magnetic field in the central plasma sheet (solid line) and plasma sheet boundary layer (dashed line). The elevation angles are angles from the GSM- $X Y$ plane $\left(90^{\circ}\right.$ is the direction of the GSM- $Z$ axis). The number of bi-directional electron events used for the calculation of the occurrence rate is given.

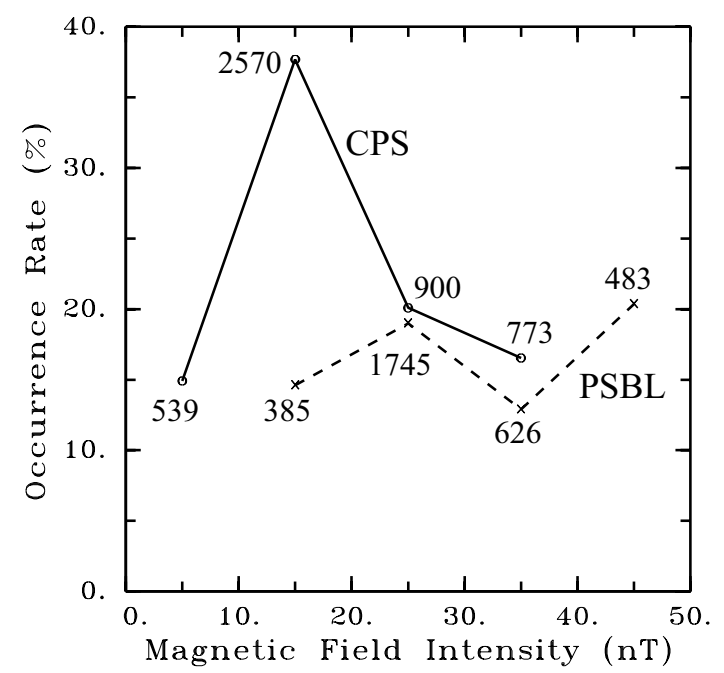

Fig. 9. Occurrence rate of bi-directional electrons at different local magnetic field intensity in the central plasma sheet (solid line) and plasma sheet boundary layer (dashed line). The number of bidirectional electron events used for the calculation of the occurrence rate is given.

It is very interesting to note that both in the CPS and in the PSBL, the occurrence rate increases as $\theta$ becomes larger.

The dependence on the magnetic field intensity is shown in Fig. 9. The highest occurrence of bi-directional electrons in the CPS is observed at $B \sim 15 \mathrm{nT}$. It decreases to both weaker and stronger magnetic fields from this value. The occurrence in the PSBL seems to be consistent with that in the CPS at the larger magnetic field region.

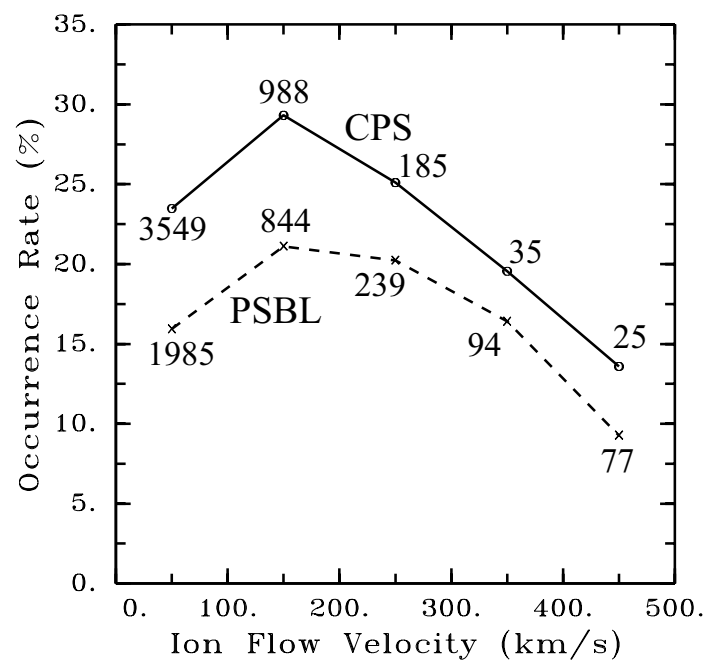

Fig. 10. Occurrence rate of bi-directional electrons at different ion flow velocity in the central plasma sheet (solid line) and plasma sheet boundary layer (dashed line). The data points at $400-500 \mathrm{~km} / \mathrm{s}$ indicate those at velocities higher than $400 \mathrm{~km} / \mathrm{s}$. The number of bidirectional electron events used for the calculation of the occurrence rate is given.

\subsection{High-speed ion flow in the CPS}

As shown in the example of Fig. 1, bi-directional electrons are observed after the passage of high-speed ion flow in the CPS region. Earthward high-speed ion flows are often observed in the near-Earth central plasma sheet (Baumjohann et al., 1990; Angelopoulos et al., 1992, 1994). We check the dependence of the bi-directional electron occurrence on the ion bulk flow velocity, $v$, in Fig. 10. Both in the CPS and PSBL, maximum occurrence is observed for flows of $v=100-200 \mathrm{~km} / \mathrm{s}$. The occurrence rate decreases as the flow velocity becomes high. This result seems to be consistent with the example of Fig. 1 , in which the ratio $F_{\|} / F_{\perp}$ tends to be smaller during the high-speed flow compared with that after the flow, and is often less than 1.5.

Figure 11 shows the bi-directional electron occurrence rate and $F_{\|} / F_{\perp}$, before and after high-speed ion flow in the CPS region. In this analysis, we defined intervals of $0-20 \mathrm{~min}$, 20-40 min, and 40-60 min, respectively, after a high-speed ion flow event with velocities of more than $400 \mathrm{~km} / \mathrm{s}$. Note that most of the observed high-speed flows are earthward. We also give data for time intervals during which no highspeed flows $(v>400 \mathrm{~km} / \mathrm{s})$ are observed for more than 60 min. Data from the PSBL are omitted in this analysis. Time intervals with high-speed flows are not included in this analysis. It is clearly seen that the occurrence rate of bidirectional electrons maximizes within $20 \mathrm{~min}$ after the highspeed flow passage. The ratio $F_{\|} / F_{\perp}$ shows a high value of 2.3 in this interval. Note that a substantial occurrence $(\sim 25 \%)$ is also seen for intervals without high-speed flow. 


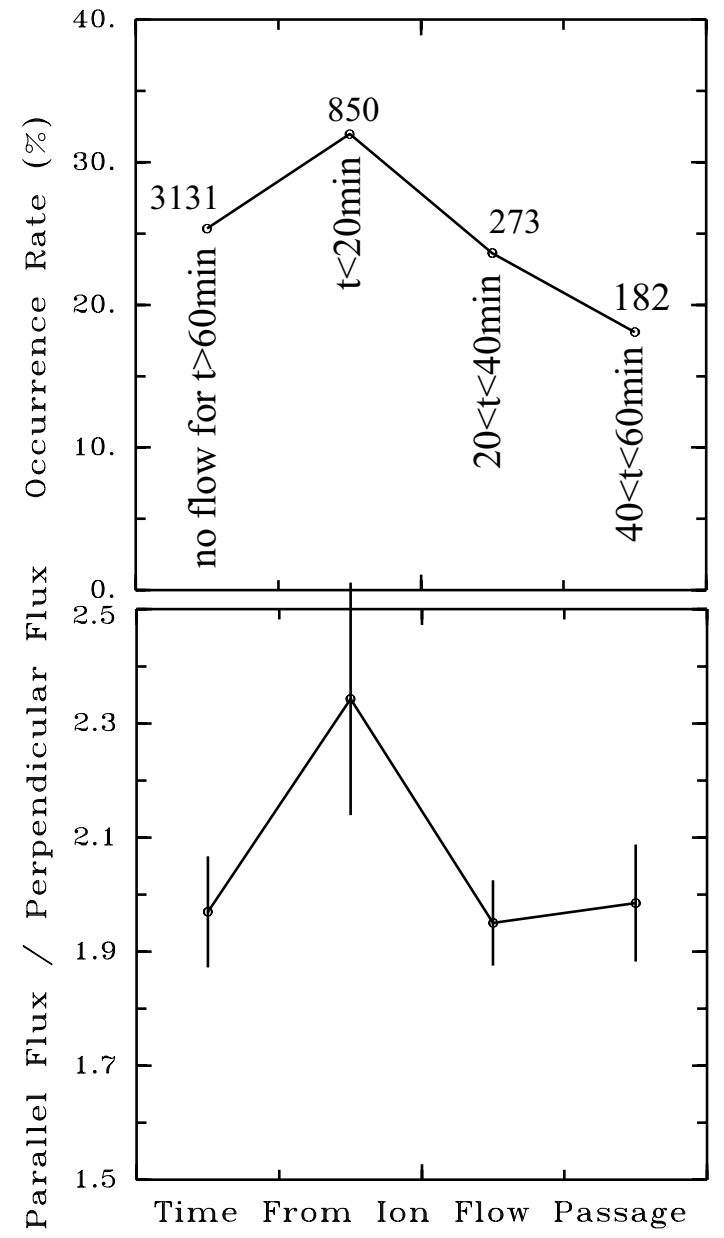

Fig. 11. (Top) occurrence rate and (bottom) ratio of parallel to perpendicular electron energy fluxes for bi-directional electron events observed in the central plasma sheet for 0-20, 20-40, and 40$60 \mathrm{~min}$ after the high-speed ion flow passage. The values at the time of no high-speed flow for $60 \mathrm{~min}$ are also indicated. The number of bi-directional electron events used for the calculation of the occurrence rate is given in the top panel. Error bars in the bottom panel represent $\pm 0.2 \sigma$. The high-speed ion flow is defined as flow with velocities in excess of $400 \mathrm{~km} / \mathrm{s}$ in the central plasma sheet.

\section{Discussion}

There are two possible sources which produce bi-directional electrons. One source is electrons from the ionosphere, while the other is some acceleration process in the plasma sheet. If electrons come up from the ionosphere, they become a highly collimated beam in the regions of weak magnetic field to conserve the first adiabatic invariant. Assuming that the magnetic field intensity at the ionospheric altitude is $\sim 50000 \mathrm{nT}$, the electrons become a beam with pitch angles less than $2.6^{\circ}$ at the point where the magnetic field intensity is $100 \mathrm{nT}$. This means that the electrons which come up from the ionosphere are already highly collimated in the region of $B \sim 100 \mathrm{nT}$. However, our observation shows that the occurrence rate of bi-directional electrons drastically decreases in the CPS as the magnetic field intensity increases from $15 \mathrm{nT}$ to $35 \mathrm{nT}$
(Fig. 9). This fact is not consistent with a source of bidirectional electrons in the ionosphere.

On the other hand, the present results seem to be consistent with the production of the bi-directional electrons in the vicinity of the neutral sheet, where the field intensity becomes minimum. Other observational facts, i.e. the high occurrence of bi-directional electrons in the inner CPS region (Fig. 5), in large- $\theta$ regions (Fig. 8), and in large- $\left|X_{\mathrm{GSM}}\right|$ regions (Fig. 6a), also suggest that bi-directional electrons are produced near the neutral sheet.

Hada et al. (1981), Smets et al. (1999), and Sergeev et al. (2001) have suggested the production of bi-directional electrons by Fermi-type acceleration near the neutral sheet. To create the acceleration energy, it is necessary to consider a dusk-to-dawn electric field, which is equivalent to earthward plasma flow perpendicular to the northward magnetic field. It is quite likely that the bi-directional electrons observed after the earthward high-speed flow passage are produced by the Fermi-type electron acceleration.

It should be noted, however, that the occurrence rate of high-speed flow of $(v>400 \mathrm{~km} / \mathrm{s})$ is only $\sim 1-4 \%$ of the whole observation in this tail region (e.g. Shiokawa et al., 1997; Angelopoulos et al., 1994). As shown by Baumjohann et al. (1989) and Hori et al. (2000), the ion flow velocity in this region is usually less than $100 \mathrm{~km} / \mathrm{s}$, even for the active time of $A E>100 \mathrm{nT}$. Because the average flow in this region is mostly earthward, the Fermi-type acceleration may occur not only associated with the high-speed flow of $v>400 \mathrm{~km} / \mathrm{s}$, but also during such prevailing slow earthward flows in the tail.

The Fermi-type acceleration of electrons can be analytically expressed by defining the energy anisotropy $A_{w}=$ $W_{\|} / W_{\perp}$, where $W_{\|}$and $W_{\perp}$ are average energies parallel and perpendicular to the magnetic field, respectively. When electrons move inward in the tail from area 1 to area 2 by, for example, $\mathbf{E} \times \mathbf{B}$ drift of duskward electric field, the anisotropy change from $A_{w 1}$ to $A_{w 2}$ by the Fermi acceleration is given as (Baumjohann and Treumann, 1996),

$\frac{A_{w 2}}{A_{w 1}}=\frac{B_{1}}{B_{2}} \frac{l_{1}^{2}}{l_{2}^{2}}$,

where $B$ and $l$ are magnetic field intensity and field-line length, respectively, and the suffixes 1 and 2 indicate values at area 1 and 2 , respectively. Because $B_{1}<B_{2}$ and $l_{1}>l_{2}$, the parallel acceleration occurs only if the square of the field line length decreases less than the magnetic field increase.

Equation (1) seems to support our observational result of the tailward increase of bi-directional electrons in CPS (Fig. 6). Namely, in the region close to $18 R_{E}$, the field line will be in a tail-like form, where radial change in $B$ is much smaller than that of $l^{2}$, and therefore, the parallel acceleration will occur. On the other hand, in the region around $10 R_{E}$, the field line will be much more dipolar, where the radial change of $B$ is larger and that of $l^{2}$ is smaller. Also, as shown by Hori et al. (2000), the earthward flow velocity increases with increasing the radial distance. 
It should be noted that our results do not exclude other mechanisms that produce bi-directional electrons in the vicinity of the neutral sheet, e.g. parallel electron acceleration by turbulent fields or plasma waves. A substantial amount of bi-directional electrons is observed during time intervals without high-speed ion flow (Fig. 11) and under fairly quiet conditions (Fig. 2).

It should also be noted that our results do not entirely exclude the possibility of ionospheric source to form the bi-directional electrons. There is a continuous occurrence of bi-directional electrons of $15-20 \%$ for all $B$ regions in Fig. 9 in CPS. The occurrence is not zero but $\sim 10 \%$ around $\left|X_{\mathrm{GSM}}\right|=10 R_{E}$ in CPS, as shown in Fig. 6. It is possible to relate part of these 10-20\% occurrences to the ionospheric source.

As discussed above, electrons from the ionosphere form sharply field-aligned beams in the magnetosphere due to the conservation of the magnetic moment. In our analysis, we took the flux ratio $F_{\|} / F_{\perp}>1.5$, which was taken also by Klumpar (1993), for defining bi-directional electrons. The flux ratio used recently for the definitions of highlycollimated bi-directional electrons is $\sim 4.0$ (Sugiyama et al., 1997; Fujimoto et al., 1997) per each energy channel. Most of the bi-directional electron events selected in this paper have a relatively weak pitch angle anisotropy, because about $80 \%$ of the bi-directional electron events have a flux ratio between 1.5 and 2 . If we use a flux ratio of more than 3 , the total number of bi-directional electron events in the CPS is 274 , which is not enough to discuss occurrence characteristics. Although the integration of all energy bins tends to reduce the flux ratio, we note that our result of occurrence rate does not focus on the highly collimated electron beams, due to our limited data set.

As shown by Baumjohann et al. (1988, 1989, 1991), the particle fluxes and energies in the plasma sheet are highly variable depending on magnetic activity (more explicitly on substorm phase), radial distance, and distance from the neutral sheet. The large standard deviations of energies and fluxes in Figs. 5, 7, and 11 are mainly due to these variations.

In the PSBL, the occurrence characteristics of bidirectional electrons are different from those in the CPS. The occurrence is almost constant, independent from magnetic field intensity, for $15-45 \mathrm{nT}$, and radial distance for $-19<X_{\mathrm{GSM}}<-9 R_{E}$, as shown in Figs. 8 and 6a, respectively. The occurrence peak around midnight is much weaker in the PSBL than in the CPS (Fig. 6b). Takahashi and Hones (1988) have suggested that the PSBL is connected to a neutral line which is located tailward of the satellite. The bi-directional electrons in the PSBL may be generated by the reconnection process at the neutral line and the mirroring motion due to the converging magnetic field near the Earth. For most of the time intervals analyzed, the neutral line is probably located in the distant tail $\left(X_{\mathrm{GSM}}>100 R_{E}\right)$ (e.g. Nishida et al., 1996), since the near-Earth neutral line is formed only for the short interval of the substorm expansion phase. The observed characteristics of bi-directional electrons in the PSBL seem to be consistent with the idea that the bi-directional electrons in the PSBL are produced near the distant neutral line.

It should be noted that there is a second peak of the occurrence rate of bi-directional electrons in the outermost layers of the CPS, within 3 min to the PSBL entry (Fig. 5). As shown in the example in Fig. 3, these electrons seem to occur inside (plasma sheet side) of the PSBL ion flow. This fact is not consistent with the view by Takahashi and Hones (1988), since a dawn-to-dusk electric field which pushes particles into the plasma sheet acts more effectively on ions than on electrons. Nagatsuma et al. (1995) have pointed out that there are low energy electron bursts just equatorward of the PSBL ion signature on the basis of particle observation by the Akebono satellite at the ionospheric altitude. These bursts seem to be caused by Alfvén wave resonance of particles between the ionosphere and the magnetosphere. The second peak of the bi-directional electron occurrence discussed here may be the magnetospheric counterpart of these electron bursts.

\section{Conclusions}

We have studied the occurrence characteristics of fieldaligned bi-directional electrons with $F_{\|} / F_{\perp}>1.5$ observed by the AMPTE/IRM satellite in the near-Earth plasma sheet at $\left|X_{\mathrm{GSM}}\right|=9-19 R_{E}$. The observed characteristics are summarized as follows.

1. In the CPS, the occurrence rate of bi-directional electrons increases for larger $X_{\mathrm{GSM}}$ distance and for larger elevation angles of the magnetic field. It peaks for magnetic field intensities of $\sim 15 \mathrm{nT}$ and near midnight $\left(Y_{\mathrm{GSM}} \sim 0\right)$. Hence, bi-directional electrons are most frequently observed in the vicinity of the midnight sector neutral sheet.

2. In the CPS, bi-directional electrons are intensified after the high-speed ion flow passage.

3. Both in the CPS and in the PSBL, the occurrence rate of bi-directional electrons increases with increasing magnetic activity.

4. There are some clear bi-directional electron events in the CPS region, even when magnetic activity and plasma sheet activity are fairly low (e.g. Fig. 2).

5. In the PSBL region, the occurrence is almost constant, independent from magnetic field intensity and $X_{\mathrm{GSM}}$ distance.

6. There is a small peak of the bi-directional electron occurrence rate in the outermost layers of the CPS within 3 min to the PSBL entry.

From these observational facts we conclude that the major source of bi-directional electrons in the CPS lies in the vicinity of the neutral sheet, including Fermi acceleration, and the contribution from ionospheric electrons is minor in this tail region. The bi-directional electron features in the PSBL 
are consistent with bi-directional electron generation near the distant neutral line. The occurrence characteristics of highlycollimated bi-directional electrons with $F_{\|} / F_{\perp}>\sim 4$ are still an open question.

Acknowledgements. We are grateful to the late N. Sckopke for his kind support in accessing the AMPTE/IRM particle distribution functions. Hermann Lühr is the Principal Investigator of the fluxgate magnetometer aboard AMPTE/IRM. K. S. is grateful to the Max-Planck-Institut für extraterrestrische Physik in Garching, Germany for their hospitality and support during his one-year stay from July 1996 to June 1997. His stay was supported by the Ministry of Education, Science, and Culture of Japan with a Grant-in-Aid for Overseas Research Scholar (8-Wakate-70).

Topical Editor G. Chanteur thanks D. Delcourt and two other referees for their help in evaluating this paper.

\section{References}

Angelopoulos, V., Baumjohann, W., Kennel, C. F., Coroniti, F. V., Kivelson, M. G., Pellat, R., Walker, R. J., Lühr, H., and Paschmann, G.: Bursty bulk flows in the inner central plasma sheet, J. Geophys. Res., 97, 4027-4039, 1992.

Angelopoulos, V., Kennel, C. F., Coroniti, F. V., Pellat, R., Kivelson, M. G., Walker, R. J., Russell, C. T., Baumjohann, W., Feldman, W. C., and Gosling, J. T.: Statistical characteristics of bursty bulk flow events, J. Geophys. Res., 99, 21 257-21 280, 1994.

Baumjohann, W., Paschmann, G., Sckopke, N., Cattell, C. A., and Carlson, C. W.: Average ion moments in the plasma sheet boundary layer, J. Geophys. Res., 93, 11 507-11 520, 1988.

Baumjohann, W., Paschmann, G., and Cattell, C. A.: Average plasma properties in the central plasma sheet, J. Geophys. Res., 94, 6597-6606, 1989.

Baumjohann, W., Paschmann, G., and Lühr, H.: Characteristics of high-speed ion flows in the plasma sheet, J. Geophys. Res., 95, 3801-3809, 1990.

Baumjohann, W., Paschmann, G., Nagai, T., and Lühr, H.: Superposed epoch analysis of the substorm plasma sheet, J. Geophys. Res., 96, 11 605-11 608, 1991.

Baumjohann, W. and Treumann, R. A.: Basic space plasma physics, Imperial Colledge Press, London, 1996.

Eastman, T. E., Frank, L. A., Peterson, W. K., and Lennartsson, W.: The plasma sheet boundary layer, J. Geophys. Res., 89, 15531572, 1984.

Eastman, T. E., Frank, L. A., and Huang, C. Y.: The boundary layers as the primary transport regions of the Earth's magnetotail, J. Geophys. Res., 90, 9541-9560, 1985.

Fairfield, D. H.: A statistical determination of the shape and position of the geomagnetic neutral sheet, J. Geophys. Res., 85,
775-780, 1980.

Fujimoto, M., Sugiyama, T., Mukai, T., Saito, Y., Yamamoto, T., and Kokubun, S.: Highly collimated electron beams in the dawnflank of the magnetotail, Phys. Chem. Earth, 22, 645-651, 1997.

Hada, T., Nishida, A., Terasawa, T., and Hones, Jr., E. W.: Bidirectional electron pitch angle anisotropy in the plasma sheet, J. Geophys. Res., 86, 11 211-11 224, 1981.

Hori, T., Maezawa, K., Saito, Y., and Mukai, T.: Average profile of ion flow and convection electric field in the near-Earth plasma sheet, Geophys. Res. Lett., 27, 1623-1626, 2000.

Klumpar, D. M.: Statistical distributions of the auroral electron albedo in the magnetosphere, in: Auroral Plasma Dynamics, Geophys. Monogr. Ser., 80, (Ed) Lysak, R. L., pp. 163-171, AGU, Washington, D. C., 1993.

Lühr, H., Klocker, N., Oelschlagel, B., Hausler, B., and Acuña, M.: The IRM fluxgate magnetometer, IEEE Trans. Geosci. Remote Sens., 23, 259-261, 1985.

Nagatsuma, T., Fukunishi, H., and Mukai, T.: Spatial relationships between field-aligned currents and suprathermal electron beams observed at the poleward boundary of the nightside auroral oval, J. Geophys. Res., 100, 1625-1637, 1995.

Nishida, A., Mukai, T., Yamamoto, T., Saito, Y., and Kokubun, S.: Magnetotail convection in geomagnetically active times 1 . Distance to the neutral lines, J. Geomag. Geoelectr., 48, 489-501, 1996.

Paschmann, G., Loidl, H., Obermayer, P., Ertl, M., Laborenz, R., Sckopke, N., Baumjohann, W., Carlson, C. W., and Curtis, D. W.: The plasma instrument for AMPTE/IRM, IEEE Trans. Geosci. Remote Sens., 23, 262-266, 1985.

Sergeev, V. A., Baumjohann, W., and Shiokawa, K.: Bi-directional electron distributions associated with near-tail flux transport, Geophys. Res. Lett., 28, 3813-3816, 2001.

Shiokawa, K., Baumjohann, W., and Haerendel, G.: Braking of high-speed flows in the near-Earth tail, Geophys. Res. Lett., 24, 1179-1182, 1997.

Shiokawa, K., Baumjohann, W., Haerendel, G., Paschmann, G., Fennell, J. F., Friis-Christensen, E., Lühr, H., Reeves, G. D., Russell, C. T., Sutcliffe, P. R., and Takahashi, K.: High-speed ion flow, substorm current wedge, and multiple Pi2 pulsations, J. Geophys. Res., 103, 4491-4507, 1998.

Smets, R., Delcourt, D., Sauvaud, J. A., and Koperski, P.: Electron pitch angle distributions following the dipolarization phase of a substorm: Interball-Tail observations and modeling, J. Geophys. Res., 104, 14 571-14 581, 1999.

Sugiyama, T., Fujimoto, M., Nagai, T., Mukai, T., Saito, Y., Terasawa, T., Yamamoto, T., and Kokubun, S.: Highly collimated electron beams observed during quiet times, Geophys. Res. Lett., 24, 1651-1654, 1997.

Takahashi, K. and Hones, Jr., E. W.: ISEE 1 and 2 observations of ion distributions at the plasma sheet-tail lobe boundary, J. Geophys. Res., 93, 8558-8582, 1988. 